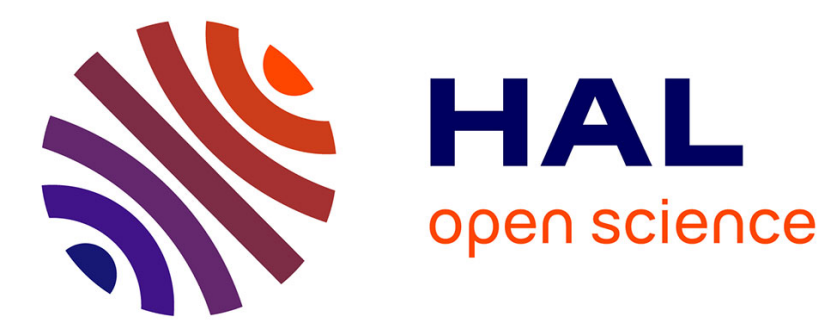

\title{
Collaborative Oscillatory Fracture
}

Juan-Francisco Fuentealba, Joel Marthelot, Benoit Roman, Francisco Melo

\section{To cite this version:}

Juan-Francisco Fuentealba, Joel Marthelot, Benoit Roman, Francisco Melo. Collaborative Oscillatory Fracture. Physical Review Letters, 2020, 10.1103/PhysRevLett.124.174102 . hal-02973911

\section{HAL Id: hal-02973911 \\ https://hal.science/hal-02973911}

Submitted on 21 Oct 2020

HAL is a multi-disciplinary open access archive for the deposit and dissemination of scientific research documents, whether they are published or not. The documents may come from teaching and research institutions in France or abroad, or from public or private research centers.
L'archive ouverte pluridisciplinaire HAL, est destinée au dépôt et à la diffusion de documents scientifiques de niveau recherche, publiés ou non, émanant des établissements d'enseignement et de recherche français ou étrangers, des laboratoires publics ou privés. 


\title{
Collaborative Oscillatory Fracture
}

\author{
Juan-Francisco Fuentealba $\odot,{ }^{1, *}$ Joel Marthelot $\odot,{ }^{2}$ Benoît Roman $\odot,{ }^{3}$ and Francisco Melo $\oplus^{1,4}$ \\ ${ }^{1}$ Departamento de Física Universidad de Santiago de Chile, Avenida Ecuador 3493, 9170124 Estación Central, Santiago, Chile \\ ${ }^{2}$ Aix-Marseille Univ., CNRS, IUSTI, 13013 Marseille, France \\ ${ }^{3}$ PMMH, CNRS, ESPCI Paris, Université PSL, Sorbonne Université, Université de Paris, F-75005, Paris, France \\ ${ }^{4}$ SMAT-C, Universidad de Santiago, Avenida B. O'Higgins 3363, Santiago-Chile
}

(Received 17 January 2020; accepted 23 March 2020; published 30 April 2020)

\begin{abstract}
We report a new oscillatory propagation of cracks in thin films where three cracks interact mediated by two delamination fronts. Experimental observations indicate that delamination fronts joining the middle crack to the lateral crack tips swap contact periodically with the crack tip of the middle crack. A model based on a variational approach analytically predicts the condition of propagation and geometrical features of three parallel cracks. The stability conditions and oscillating propagation are found numerically and the predictions are in favorable agreement with experiments. We found that the physical mechanism selecting the wavelength structure is a relaxation process in which the middle crack produces a regular oscillatory path.
\end{abstract}

DOI: 10.1103/PhysRevLett.124.174102

Fracture propagation in thin films resulting from residual tensile stresses is common in everyday life and detrimental in numerous practical applications including protective coating, multilayered devices [1], and art [2]. By contrast, crack propagation has recently been envisioned as a tool to create patterns in thin films and tailor surface microstructures [3,4]. Oscillatory cracks, observed in thermally quenched glass $[5,6]$, tearing through thin sheets $[7,8]$, or channel cracks in thin films [3,9] have stimulated a number of theoretical and numerical studies [10-14]. In this Letter, we report a new oscillating propagation in thin layers when three cracks interact with two delamination fronts. We show that the propagation of several cracks interacting with limited delamination can be predicted computing the maximum energy release rate, based on simplifying approximation on the dependence of the form of the energy with the geometry of the delamination front. Our model accounts well for the main geometrical features of observed structures. Detailed analysis of energy released of each crack progression shows that the physical mechanism ruling the wavelength structure is a relaxation process.

Experimental observations. - Commercial organosilicate coating [spin on glass (SOG), Accuglass T-12B, Honeywell] was spin-coated on silicon wafer to produce uniform thin films with micrometric thicknesses $(h \sim[1.1-3.5] \mu \mathrm{m})$. The adhesion energy with the substrate $\Gamma_{0}$, the residual biaxial stresses $\sigma$, and the fracture properties of the thin films were characterized in [15]. Crack trajectories are followed through a microscope in reflection mode. In the presence of a moderate adhesion $\left(\Gamma_{0} \sim[0.3-1.3] \mathrm{J} / \mathrm{m}^{2}\right)$, residual biaxial stresses leads to striking regular structures [15] involving simultaneous propagation of cracks and delamination fronts.
In Fig. 1(a) and movie S1 in the Supplemental Material [16], we report a new mode of oscillatory crack propagation where three cracks interact mediated by two delamination fronts. The central crack follows a nearly sinusoidal trajectory. Delamination at each side of the middle crack is evidenced thanks to Newton's interference patterns. The delamination fronts do not reach the central crack tip simultaneously [inset of Fig. 1(b)], but instead, swap contact with the middle-crack tip. We note $s_{+}$(respectively, $s_{-}$) the curvilinear abscissa of the point where the upper (lower) debonding front reaches the central cut. At a given time [top left inset of Fig. 1(b)], the lower front reaches the center crack and will be called the "driver" front. Such propagation of a delamination front joining two advancing cracks ("crack duos") leads to a steady solution $[15,20]$ when isolated. The upper front is retarded with respect to the crack tip $\left(s_{-}<s_{+}\right)$. This situation where a single crack (here, the top one) is driven by a delamination front following an existing crack path ("follower crack") also evolves into a steady solution $[15,20]$ when the preexisting cut is infinite (we review, in the Supplemental Material [16], the salient features of asymptotic solutions for follower and duo). But here, some time later [bottom right inset on Fig. 1(b)], the role of the upper and lower fronts have inverted $\left(s_{+}>s_{-}\right)$; the upper front has become the driving point (forming a crack duo) and the lower front corresponds to a follower fracture. Figure 1(c) presents the measured distance between the driving and following points $\Delta s=\left|s_{+}-s_{-}\right|$, revealing the periodicity of the alternating dynamics between duo and follower.

The use of powerful linear elastic fracture mechanics (LEFM), i.e., the study of the stress intensity factors along the crack front, would be very difficult here: the fracture 
(a)

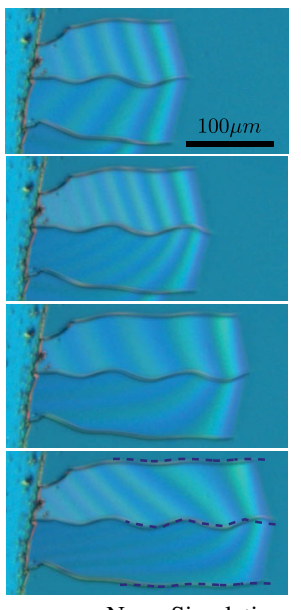

- - - Num. Simulation

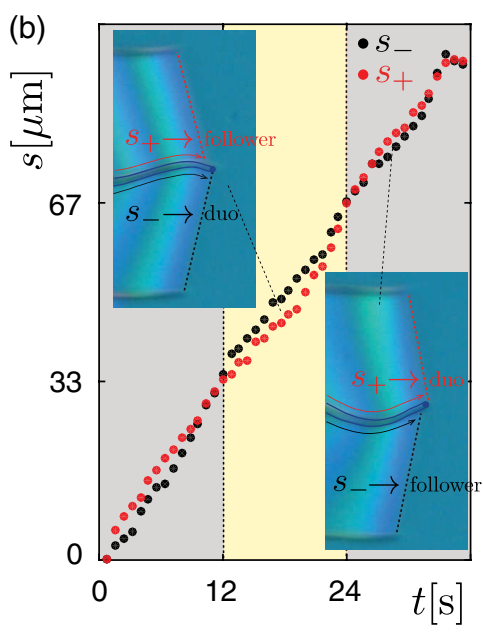

(c)

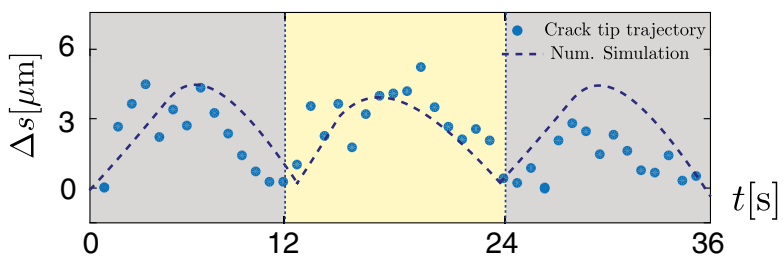

FIG. 1. (a) Snapshots of four crack configurations taken at equally spaced time intervals, $\Delta t=11 \mathrm{~s}$, illustrating middle crack oscillation. (b) Positions of ends of delamination fronts reaching the middle crack, $s_{+}$and $s_{-}$, measured along the middlecrack path as defined in the insets. (c) Position of the driver point $s_{+}$with respect to the follower point $s_{-}$. Dashed blue line in (c) shows numerical solution and dashed blue lines on the lowest panel in (a) depict the pattern obtained from numerical solution, for $\gamma e / G_{c}=0.49$ and $e / \Gamma_{0}=1.44$. Adjusting the period of oscillation to that of the experiment leads to $\eta \approx 4.5 \mu \mathrm{m} / \mathrm{s}$.

front in a thin film does not generally have a simple geometry and includes corner singularities (where stress intensity factors cannot be defined) at the surface of the film [21]. Similarly, the debonding front has no reason to be a straight line and is probably under nonuniform modemixity [1]. Here, we choose to use a variational approach, instead [22], based on direct calculation of the global energy released during propagation, an approach equivalent to LEFM (indeed, if the trajectory of the crack does not involve kinks, the principle of local symmetry is strictly equivalent to the criterion of maximal energy release rate [23]). We also use simplifying approximations [15,20], where the fracture energy only depends on the geometry of the crack path and of the delaminated area (neglecting the effect of mode mixity on energy dissipation). We will compute the conditions for propagation for all cracks (Griffith criterion), assume that they propagate in the direction that maximizes the energy release rate, and determine their individual speed. We obtain explicit propagation rules and analytically and numerically study their behavior. The mechanism of oscillation of the center crack involves delamination-front ends that may join the central crack tip either simultaneously or alternatively, as observed experimentally. In the following, we demonstrate that, if both delamination fronts join the central crack tip simultaneously, a stationary solution of three parallel cracks exists. The limits of the stability of this solution are explored in the phase diagram defined by the main independent parameters of the problem.

A model for coupled fracture and debonding.-Before any fracture occurs, the film is under isotropic uniform residual stress $\sigma$, corresponding to an elastic energy $e=$ $h \sigma^{2}(1-\nu) / E$ per unit of film area, where $\nu$ is the Poisson ratio, and $E$ the Young modulus. We assume that, when a cut with a length $s$ bounds a delaminated surface $A$ defining a straight delamination front with length $l$, the energy difference with respect to the pristine state is $[15,20]$

$$
\mathcal{E}_{r}=e A+e f(l)+\gamma e h s,
$$

The first term in this expression would represent a complete release of stress in the delaminated region, therefore, proportional to its area $A$. But stresses parallel to the debonding front remain on the order of $\sigma$ in the debonded region close to the debonding front with an area of order $l^{2}$, with an energy cost $\propto e l^{2}$. The tilting of the debonded flap along its front provides an extra energy gain $\alpha$ elh so that $f(l)=\beta l h-\alpha l^{2}$, with $\alpha$ and $\beta$ dimensionless constants. Finally, the term rehs accounts for the elastic energy released along the crack edge outside of the delamination area $(\gamma=0.64$ was numerically computed for simple channel cracks $[1,15])$.

We apply these assumptions to the case of three interacting cracks, as in Fig. 2, whose curvilinear position along their trajectories are noted $s_{+}, S_{+}, S_{-}$. Here, we have considered the case of the lower debonding front lagging behind at position $s_{-}$-in the opposite case where $s_{+}$is lagging, it suffices to exchange + and - in Eqs. (6)-(7). Griffith's criterion, accounting for energy conservation, indicates that, during propagation, the elastic energy released $d \mathcal{E}_{r}$ compensates the expense of propagating cracks and delamination

$$
d \mathcal{E}_{r}=d(\Gamma A)+G_{c} h\left(d s_{+}+d S_{+}+d S_{-}\right),
$$

where $G_{c}$ is the fracture energy in the film and $\Gamma$ the adhesion energy. We assume, for the sake of simplicity, that fracture energy $G_{c}$ is a constant, but that the adhesion energy $\Gamma$ depends linearly on the velocity $v$ of the front

$$
\Gamma(v)=\Gamma_{0}(1+v / \eta),
$$

where $\eta$ is a kinetic coefficient, accounting for energy dissipation, and $\Gamma_{0}$ is the delamination energy at vanishing speed. The debonding front, being driven at a weak interface, is subject to (nonuniform) mode mixity, modifying the debonding energy [1], but our simplified approach uses an effective spatially averaged debonding 
(a)

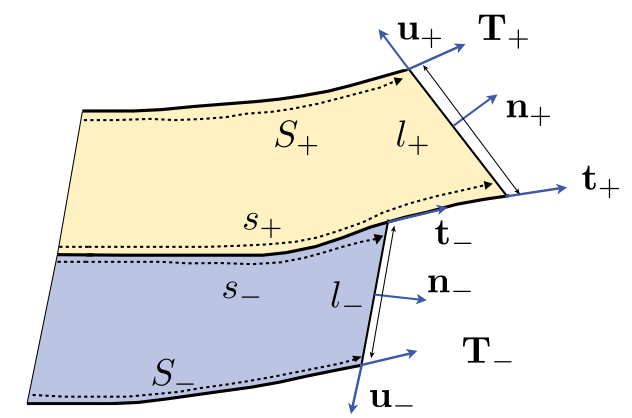

(b)

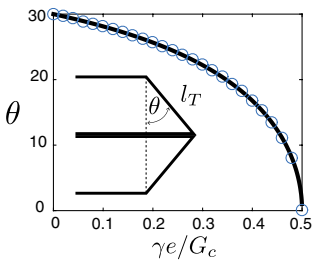

FIG. 2. (a) Sketch of the three cracks. $S_{ \pm}$are the positions of the driving and following cracks that move together with their respective delamination fronts. $s_{ \pm}$stand for the positions of the front ends reaching the middle crack. $\mathbf{T}_{ \pm}$and $\mathbf{t}_{ \pm}$are auxiliary vectors describing crack directions, whereas $\mathbf{n}_{ \pm}$and $\mathbf{u}_{ \pm}$are normal and tangent vectors to the driving (+) and lower (-) debonding fronts. (b) and (c) Black lines represent analytical predictions of the angle $\theta$ and the dimensionless length $l_{T} / h$ for three parallel cracks with $\gamma e / G_{c}$. Blue open circles are numerical simulation with $\gamma=0.64, h=1 \mu \mathrm{m}$, and $\eta=4.5 \mu \mathrm{m} / \mathrm{s}$.

energy only dependant on speed, assuming that the modemixity distributions are similar in propagation modes considered here. This simple description is coherent with experimental measurements of $\Gamma(v)$ (see Supplemental Material [16] where other dependences of $\Gamma$ with the velocity are also considered).

By independently varying each variable, we obtain one condition for the propagation of each crack

$$
\begin{aligned}
& \mathbf{F}_{+} \cdot \mathbf{T}_{+}+\gamma e h=G_{c} h, \\
& \mathbf{F}_{-} \cdot \mathbf{T}_{-}+\gamma e h=G_{c} h, \\
& \mathbf{f}_{+} \cdot \mathbf{t}_{+}+\gamma e h=G_{c} h,
\end{aligned}
$$

where $\mathbf{T}_{+}, \mathbf{T}_{-}$, and $\mathbf{t}_{+}$are unit vectors for the direction of propagation of the top, bottom, and center cracks. In these equations, $\quad \mathbf{F}_{ \pm}=\left[e-\Gamma\left(V_{ \pm}\right)\right] l_{ \pm} / 2 \mathbf{n}_{ \pm}+e\left(\beta h-2 \alpha l_{ \pm}\right) \mathbf{u}_{ \pm}$ and $\mathbf{f}_{ \pm}=\left[e-\Gamma\left(v_{ \pm}\right)\right] l_{ \pm} / 2 \mathbf{n}_{ \pm}-e\left(\beta h-2 \alpha l_{ \pm}\right) \mathbf{u}_{ \pm}$are two vectors associated with the variation of the delaminated zone (with $\mathbf{n}$ and $\mathbf{u}$ unit vectors normal and tangent to the delamination front as in Fig. 2). $V_{ \pm}=d S_{ \pm} / d t$ and $v_{+}=$ $d s_{+} / d t$ are the crack velocities. The left hand side of Eqs. (4)-(6) provide the energy release rate for each of the three cracks, which are expected to propagate in the direction of the maximum energy release rate [24-26]. As a result, the top, bottom, and center cracks propagate

along a direction given by vectors $\mathbf{F}_{+}, \mathbf{F}_{-}, \mathbf{f}_{+}$, respectively. Inserting these value for $\left(\mathbf{T}_{+}, \mathbf{T}_{-}, \mathbf{t}_{+}\right)$into Eqs. (4)-(6) provides the velocity of each crack, using Eq. (3).

A last variation governs the speed $\left(d s_{-} / d t\right)$ of the delamination front along the existing cut, for which the cost of the preexisting crack $2 \gamma e h$ must be subtracted from the energy balance

$$
\mathbf{f}_{-} . \mathbf{t}_{-}-\gamma e h=0,
$$

where $\mathbf{t}_{-}$is the unit vector tangent to the central cut at point $s_{-}$. Finally, Eqs. (4)-(7) provide the direction of propagation and speed for all relevant points and, therefore, the dynamics of the system.

Symmetric solution: three parallel cracks.-First, we consider the symmetric case where both fronts reach the central crack tip [as sketched in the inset of Fig. 2(b)]. The equations for the crack dynamics are obtained by taking $\mathbf{T}_{ \pm}=\mathbf{t}_{ \pm}=\mathbf{t}$ together with $s_{+}=s_{-}$and $S_{+}=S_{-}$. Equations (4)-(7) are combined into

$$
\begin{aligned}
& \mathbf{F}_{ \pm} \cdot \mathbf{t}+\gamma e h=G_{c} h, \\
& \left(\mathbf{f}_{+}+\mathbf{f}_{-}\right) \cdot \mathbf{t}=G_{c} h,
\end{aligned}
$$

where it must be enforced that all cracks have the same speed. We find the orientation of the delamination front (i.e., the angle $\theta$ between $\mathbf{n}$ and $\mathbf{t}$ ) and their length $l_{T}$ as

$$
\begin{gathered}
\cos 2 \theta=\frac{G_{c}}{2\left(G_{c}-\gamma e\right),} \\
l_{T}=\frac{\beta h}{2 \alpha}+\frac{G_{c} h}{2 \sqrt{2} \alpha e} \sqrt{\left(\frac{\gamma e}{G_{c}}-1\right)\left(\frac{\gamma e}{G_{c}}-\frac{1}{2}\right)}, \\
\Gamma=e-\frac{\sqrt{2} G_{c} h}{l_{T}} \sqrt{\left(\frac{\gamma e}{G_{c}}-1\right)\left(\frac{\gamma e}{G_{c}}-\frac{3}{2}\right)} .
\end{gathered}
$$

These analytical expressions are in good agreement with direct numerical computation [Figs. 2(b) and 2(c)]. We note that the geometry of this three-crack solution scales with the film thickness $h$, and only depends on $e / G_{c}$, but not on the adhesion energy $\Gamma_{0}$, as already observed in twocrack situations (duo and follower [15]). A phase diagram in the $\left(\gamma e / G_{c}, e / \Gamma_{0}\right)$ space representing the domain of crack propagation is presented in Fig. 3(a). Positive values of the fracture speed are required, i.e., $\Gamma \geq \Gamma_{0}$ in Eq. (12), where the equality defines the lower boundary of existence [red line in Fig. 3(a)]. The upper boundary is given by $\gamma e / G_{c}=0.5$ as deduced from Eq. (11). Therefore, we have found an analytical expression for the threecrack symmetric solution and, next, numerically study its stability.

Three crack stability: numerical simulations.-First, we investigate the stability of the symmetric three-crack solution by preparing the system in the stationary trio 


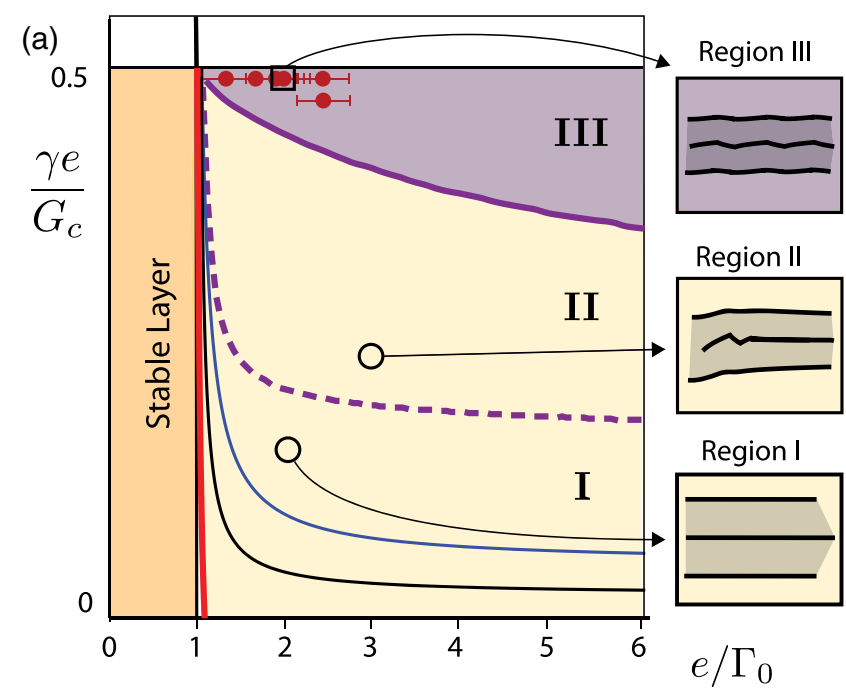

(b)
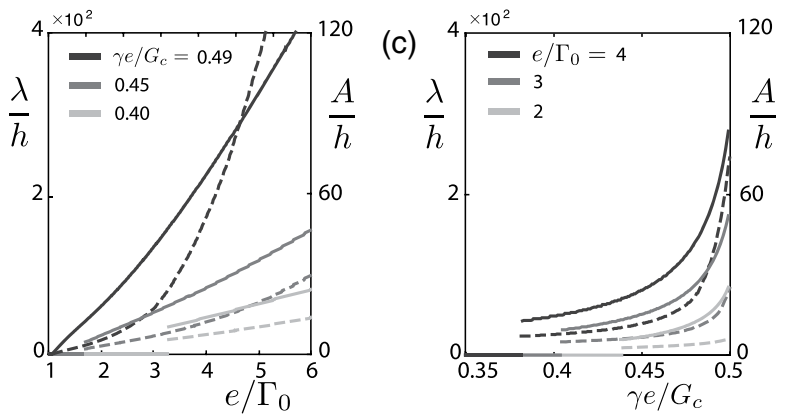

FIG. 3. (a) Phase diagram of the stable trio and its corresponding oscillatory solution. Region I (yellow): Trio propagates straight and are stable. Region II (gray): The trio stationary solution (purple inset) becomes subcritically unstable against a small delay in the delamination ends joining the central crack. Region III (purple): The trio stationary solution is unstable against a delay of delamination ends. All lines in (a) were plotted with $\gamma=0.64$. Red circles are experimental points where oscillatory trio is observed. Black open square and circles indicate the points on the phase diagram for which the right panels were obtained. Solid blue and black lines give the existence domains for duo and follower, respectively. Dimensionless wavelength, $\lambda / h$, (solid line) and dimensionless amplitude, $A / h$, (dashed line) of oscillation vs $e / \Gamma_{0}$ (b) and vs $\gamma e / G_{c}$ (c), respectively.

configuration according to Eqs. (10)-(11). Then, a very small perturbation is imposed in the form of a slight delay of one of the debonding fronts (thus, creating a follower point), and the system is allowed to evolve in time. For some conditions, this leads to an oscillatory solution. For example, for the parameter corresponding to the black square in Fig. 3, the numerical prediction of the crack path [dashed line in Figs. 1(a) and 1(c)] is found to be in good agreement with the experimental observations (where $\eta=$ $4.5 \mu \mathrm{m} \mathrm{s}^{-1}$ defining the dependence of adhesion energy with speed is the only adjusted parameter).

The time evolution of the three-crack system is solved numerically using $100 \times 100$ data points in the parameter

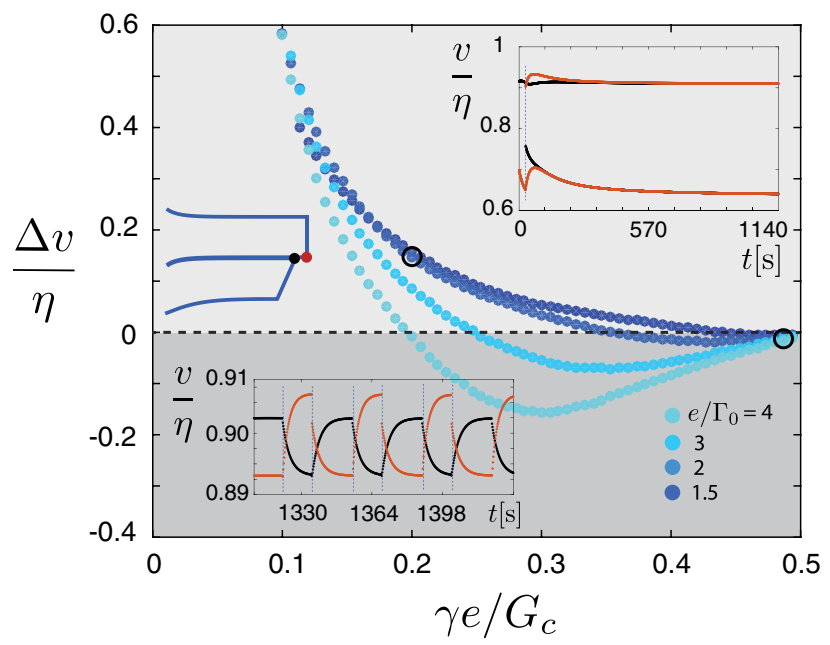

FIG. 4. Relative speed, $\Delta v$ of the following point with respect to the driving point right after the capture event. The initial condition is depicted in the left upper inset: dots indicate positions of lower (black) and upper (red) fronts on the crack path. Right upper inset: Instantaneous speeds of the following point and driving front if $\Delta v \geq 0$. Lower inset: Instantaneous speeds of front extremes on the crack trajectory as indicated on left upper inset, if $\Delta v<0$. These points periodically alternate between following and driving points; vertical dashed lines indicate capture events. Open circles indicate values used in the insets

space defined by $\gamma e / G_{c}$ and $e / \Gamma_{0}$. We find that the three crack solution is stable in regions I and II. Above the dashed purple line (region II), oscillations are observed briefly after perturbation but relax after a few oscillations. Steady oscillations are only observed in region III, a small region of the phase diagram above the solid purple line. Experimental conditions where we observed oscillating propagations are indicated with red circles in the phase diagram and are found to be in favorable agreement with numerical predictions.

In Figs. 3(b) and 3(c), we plot the amplitude and wavelength of the oscillatory solutions vs $e / \Gamma_{0}$ and $\gamma e / G_{c}$. The instability is subcritical since the amplitude jump to a finite value for both critical parameters is $\left(e / \Gamma_{0}\right)_{c}$ and $\left(\gamma e / G_{c}\right)_{c}$ [Figs. 3(b) and 3(c)]. Both amplitude and wavelength scale almost linearly with the middle crack speed $\left[v_{c} \sim\left(e / \Gamma_{0}-1\right)\right]$. However, the dependence with $\gamma e / G_{c}$ is more complex.

Instability mechanism. - Experimentally, the trio solution is typically reached starting from a pair of cracks in a duo configuration later followed by a third crack propagating parallel in the follower configuration (left upper inset in Fig. 4). Experimental observations and numerics both show that the follower front (here, lower front $s_{-}$) always advances faster than the duo driving front (upper front $s_{+}$). It eventually reaches it in a capture event, where the fronts exchange roles. They also switch the equation defining their speed from the geometry Eq. (6) $\leftrightharpoons$ Eq. (7) 
and, therefore, undergo a discontinuous jump in velocity in our simplified model.

To evidence the instability mechanism, we focus our attention in the numerics on the velocity difference $\Delta v$ between the new following front and the most advanced (driving) front immediately after the capture event (Fig. 4). If $\Delta v \geq 0$, the following point is faster than the driving point and will capture it at the next time step. The two fronts then alternate at each time step, which reveals that, in practice, they move together. This dynamics persists in time and a steady state is reached (upper right inset Fig. 4). Conversely, if $\Delta v<0$ (for larger values of $\gamma e / G_{c}$, see Fig. 4), the distance between fronts continues to increase first, but as these two fronts evolve toward their steady states, their relative speed is inverse, so that the following point is able to eventually catch the driving point. The process repeats itself into an oscillation (which either decays or is steady) that bears the features of a relaxation oscillation: a sudden acceleration at the capture point followed by a process in which both the following and driving fronts tend to a stationary propagation speed (inset in Fig. 4) finally triggering a new event.

Conclusions. - We have presented a general, simple and efficient framework based on direct computation of the global energy release rate that is able to capture the rich dynamics of multiple crack tips coupled with delamination. Such phenomena would be out of reach of the powerful tools of linear elastic fracture mechanics. The new instability mechanism leading to oscillatory fracture presented here falls in the class of oscillation-relaxation, with a strong perturbation relaxing toward equilibrium (but then triggering a new event). Such a relaxation mechanism seems to capture the main features of other oscillatory fracture problems $[7,14,15]$ where the trajectory often evidence kinks at the point of maximum amplitude, suggesting periodic strong perturbations. Thus, the situation elucidated here is the first case of fracture propagation where we can express the relaxation mechanism in a simple set of equations and which can serve as an inspiration to describe oscillatory fracture in general.

The authors are very grateful to José Bico for enlightening discussions. We acknowledge support from the Convenio de Desempeño Grant No. USA1799 and Dicyt Grant No. 041831MH-POSTDOC of Universidad de Santiago de Chile, Fondecyt Grants No. 1161010, No. 3170907. The Laboratoire International Associé "Matière: Structure et Dynamique"; LIA-MSD is kindly acknowledged for supporting this scientific collaboration. *juan.fuentealbadu@usach.cl

[1] J. W. Hutchinson and Z. Suo, Adv. Appl. Mech. 29, 63 (1992).

[2] V. Lazarus and L. Pauchard, Soft Matter 7, 2552 (2011).

[3] K. Nam, I. Park, and S. Ko, Nature (London) 485, 221 (2012).

[4] M. Kim, D.-J. Kim, D. Ha, and T. Kim, Nanoscale 8, 9461 (2016).

[5] A. Yuse and M. Sano, Nature (London) 362, 329 (1993).

[6] O. Ronsin, F. Heslot, and B. Perrin, Phys. Rev. Lett. 75, 2352 (1995).

[7] B. Audoly, P. M. Reis, and B. Roman, Phys. Rev. Lett. 95, 025502 (2005).

[8] T. Tallinen and L. Mahadevan, Phys. Rev. Lett. 107, 245502 (2011).

[9] L. Goehring, W. J. Clegg, and A. F. Routh, Soft Matter 7, 7984 (2011).

[10] M. Adda-Bedia and Y. Pomeau, Phys. Rev. E 52, 4105 (1995).

[11] M. Adda-Bedia and M. Ben Amar, Phys. Rev. Lett. 76, 1497 (1996).

[12] B. Yang and K. Ravi-Chandar, J. Mech. Phys. Solids 49, 91 (2001).

[13] H. Henry and H. Levine, Phys. Rev. Lett. 93, 105504 (2004).

[14] F. Corson, M. Abba-Bedia, H. Henry, and E. Katzav, Int. J. Fract. 158, 1 (2009).

[15] J. Marthelot, B. Roman, J. Bico, J. Teisseire, D. Dalmas, and F. Melo, Phys. Rev. Lett. 113, 085502 (2014).

[16] See Supplemental Material at http://link.aps.org/ supplemental/10.1103/PhysRevLett.124.174102 for additional theoretical and experimental details, which includes Refs. [17-19].

[17] H. M. Jensen, J. W. Hutchinson, and K.-S. Kim, Int. J. Solid Struct. 26, 1099 (1990).

[18] Y. Lin, T. Y. Tsui, and J. J. Vlassak, Acta Mater. 55, 2455 (2007).

[19] Y. Lin, T. Y. Tsui, and J. J. Vlassak, J. Electrochem. Soc. 157, G53 (2010).

[20] J. Marthelot, J. Bico, F. Melo, and B. Roman, J. Mech. Phys. Solids 84, 214 (2015).

[21] A. Kotousov, Int. J. Solids Struct. 47, 1916 (2010).

[22] G. A. Francfort and J.-J. Marigo, J. Mech. Phys. Solids 46, 1319 (1998).

[23] A. Chambolle, G. A. Francfort, and J.-J. Marigo, J. Mech. Phys. Solids 57, 1614 (2009).

[24] F. Erdogan and G. Sih, ASME J. Basic Eng. 85, 519 (1963).

[25] A. Takei, B. Roman, J. Bico, E. Hamm, and F. Melo, Phys. Rev. Lett. 110, 144301 (2013).

[26] J.-F. Fuentealba, E. Hamm, and B. Roman, Phys. Rev. Lett. 116, 165501 (2016). 